\title{
Normalization of pulmonary hypertension after experimental pulmonary denervation therapy and MitraClip implantation in a patient initially disqualified from heart transplant
}

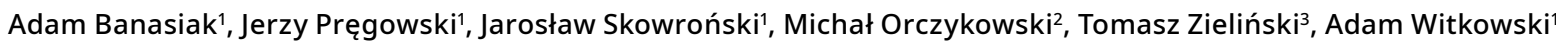 \\ 1 Department of Interventional Cardiology and Angiology, National Institute of Cardiology, Warsaw, Poland \\ 2 Department of Arrhythmia, National Institute of Cardiology, Warsaw, Poland \\ 3 Department of Heart Failure and Transplantology, National Institute of Cardiology, Warsaw, Poland
}

Correspondence to: Jerzy Pręgowski, MD, National Institute of Cardiology, ul. Alpejska 42, 04-628 Warszawa, Poland, phone: +48223434127 , email: jerzypregowski74@gmail.com Received: April 20, 2020. Revision accepted: June 22, 2020. Published online: July 6, 2020. Kardiol Pol. 2020; 78 (9): 945-946 doi:10.33963/KP.15490 Copyright by the Author(s), 2020
One of the contraindications for the orthotopic heart transplantation (OHT) is irreversible pulmonary hypertension (PH). A 62-year-old man was admitted with heart failure (HF) of ischemic etiology in NYHA class III with reduced ejection fraction (EF, 15\%), atrial fibrillation, and diabetes. Previously, the CRT was implanted. The patient underwent screening as a candidate for OHT. Echocardiography, apart from revealing impaired left ventricular contractility, showed also $\mathrm{PH}$ (right ventricle systolic pressure, $70 \mathrm{~mm} \mathrm{Hg}$ ), dilatation of the left ventricle (left ventricular end diastolic diameter, $74 \mathrm{~mm}$ ) and moderate / significant mitral regurgitation (FIGURE $1 \mathrm{~A}$ and $1 \mathrm{~B}$ ). Right heart catheterization confirmed severe $\mathrm{PH}$ : mean pulmonary artery pressure (mPAP) of $54 \mathrm{~mm} \mathrm{Hg}$, pulmonary capillary wedge pressure (PCWP) of $25 \mathrm{~mm} \mathrm{Hg}$, pulmonary vascular resistance (PVR) of 10.3 Wood units with cardiac index of $1.46 \mathrm{l} / \mathrm{min} / \mathrm{m}^{2}$. No satisfactory response to the iloprost was observed, PVR dropped to 3.6 Wood units only. Due to irreversible $\mathrm{PH}$, the patient was disqualified from OHT. Off-label sildenafil was started and routine heart failure treatment continued. During the next hospitalization 7 months later, right heart catheterization was repeated. Based on its results that confirmed persistence of $\mathrm{PH}$, the patient was enrolled into the scientific protocol of experimental transcatheter pulmonary artery denervation. The key inclusion criteria for the study were nonreversible
mPAP (not reduced $\geq 10 \mathrm{~mm} \mathrm{Hg}$ after administration of a vasodilator, iloprost) and PVR of 3.5 Wood units or more. Pulmonary artery denervation is a catheter-based ablation technique targeting afferent and efferent fibers of a baroreceptor reflex in the main pulmonary artery trunk. 'This reflex is described as responsible for elevation of the PAP in PH. Immediately before the procedure, right heart catheterization showed mPAP of $45 \mathrm{~mm} \mathrm{Hg}$ and PCWP of $19 \mathrm{~mm} \mathrm{Hg}$. After denervation, it showed mPAP of $36 \mathrm{~mm} \mathrm{Hg}$ and PCWP of $17 \mathrm{~mm} \mathrm{Hg}$. Despite hemodynamic improvement, the patient still remained in NYHA class III with exacerbation periods and was scheduled for MitraClip therapy by the Heart Team. During the MitraClip procedure, a reduction of the mitral regurgitation grade from severe to mild was achieved (FIGURE $1 \mathrm{C}$ and 1D). Optimal medical treatment including sildenafil was continued. After 4-month follow-up, right cardiac catheterization revealed a profound decrease of PH (mPAP, $36 \mathrm{~mm} \mathrm{Hg}$; PCWP, $24 \mathrm{~mm} \mathrm{Hg}$; PVR, 2.05 Wood units); the cardiac index was $3.02 \mathrm{l} / \mathrm{min} / \mathrm{m}^{2}$. Following those findings and the patient's clinical condition, the Heart Team requalified him, and successful OHT was performed 5 months later.

After surgery, the patient received a standard triple immunosuppressive regimen: tacrolimus, mycophenalate mofetil, and glucocorticoid. Despite histopathological signs of acute 

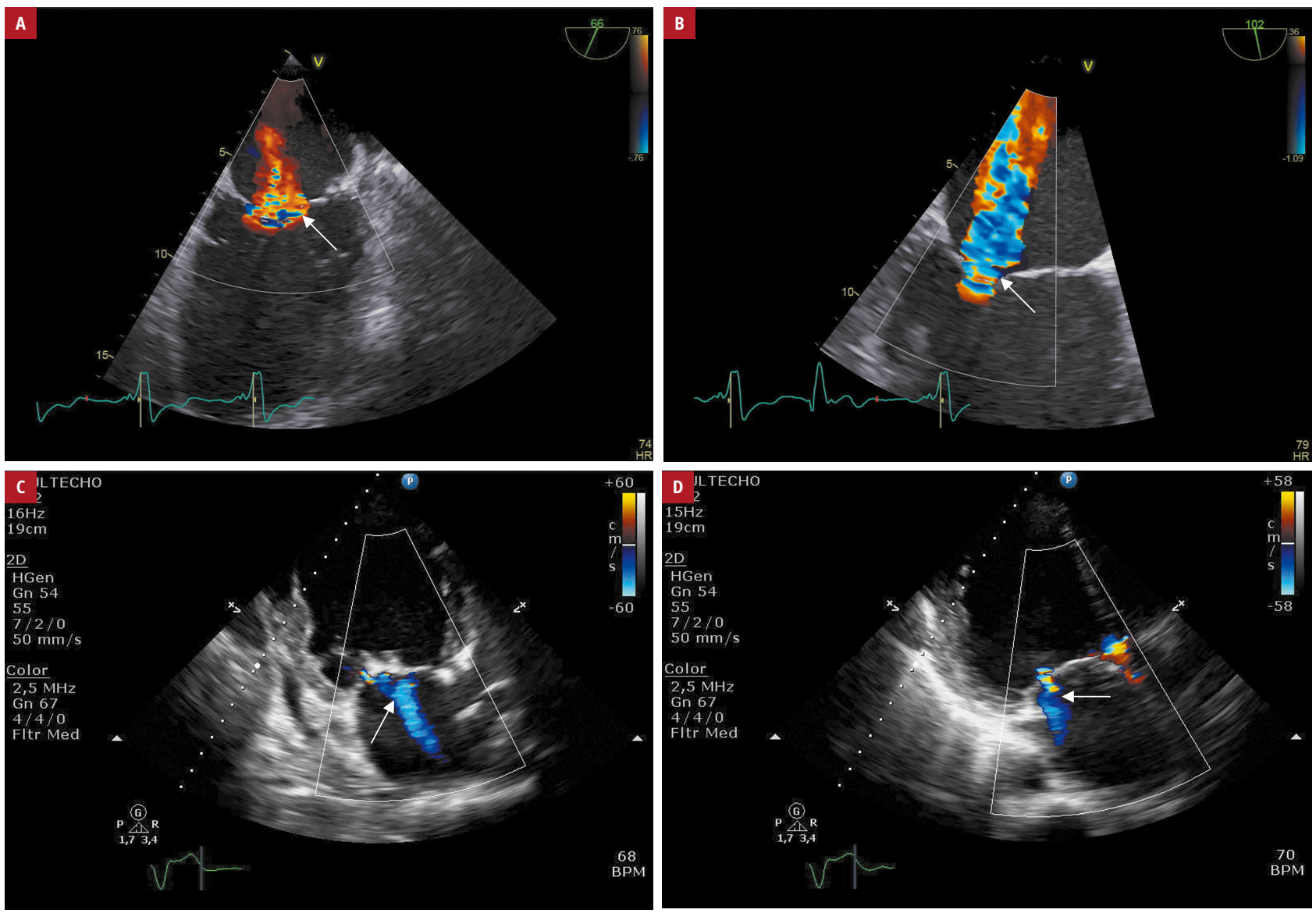

FIGURE 1 A, B - transesophageal echocardiography, X-plain view showing severe mitral regurgitation prior to the MitraClip implantation (arrows);

C, D- transthoracic echocardiography showing mild residual mitral regurgitation after implantation of 2 MitraClip devices (arrows)

rejection in the first 3 biopsies, he remained stable. Follow-up echocardiography showed slight general hypokinesis with left ventricular ejection fraction of $50 \%$.

Recently published data suggest that a successful MitraClip procedure may lead in some patients to an improvement of right ventricle function and reduction of pulmonary artery pressure. ${ }^{2}$ We believe that in this patient, the complex advanced therapy including experimental pulmonary denervation and MitraClip procedure in addition to the optimal medical treatment led to the reversal of severe $\mathrm{PH}$ and facilitated the destination life-saving therapy. However, further studies are needed to better define the role of percutaneous procedures as potential bridges facilitating OHT.

\section{ARTICLE INFORMATION}

\section{CONFLICT OF INTEREST None declared.}

OPEN ACCESS This is an Open Access article distributed under the terms of the Creative Commons Attribution-NonCommercial-NoDerivatives 4.0 International License (CC BY-NC-ND 4.0), allowing third parties to download articles and share them with others, provided the original work is properly cited, not changed in any way, distributed under the same license, and used for noncommercial purposes only. For commercial use, please contact the journal office at kardiologiapolska@ptkardio.pl.

HOW TO CITE BanasiakA, PregowskiJ, SkowrońskiJ, et al. Normalization of pulmonary hypertension after experimental pulmonary denervation therapy and MitraClip implantation in a patient initially disqualified from heart transplant. Kardiol Pol. 2020; 78: 945-946, doi:10.33963/KP.15490

\section{REFERENCES}

1 Kim CW, Aronow WS, Dutta T, et al. Pulmonary artery denervation as an innovative treatment for pulmonary hypertension with and without heart failure. Cardiol Rev. 2020 Feb 3. [Epub ahead of print].

2 Ledwoch J, Fellner C, Hoppmann P, et al. Impact of transcatheter mitral valve repair using Mitraclip on right ventricular remodeling. Int J Cardiovasc Imaging. 2020; 36: 811-819. 